\title{
CORRECTION
}

\section{Correction: 1,2,4-Trimethoxybenzene selectively inhibits NLRP3 inflammasome activation and attenuates experimental autoimmune encephalomyelitis}

Ya-jin Liao ${ }^{1,2,3,4}$, Rui-yuan Pan², Xiang-xi Kong ${ }^{2}$, Yong Cheng ${ }^{1}$, Lu Du², Zhen-chao Wang ${ }^{3}$, Chao Yuan ${ }^{2}$, Jin-bo Cheng ${ }^{1}$, Zeng-qiang Yuan ${ }^{2}$ and Hai-yan Zhang $^{3}$

Acta Pharmacologica Sinica (2022) 43:504; https://doi.org/10.1038/s41401-021-00670-z

Correction to: Acta Pharmacologica Sinica https://doi.org/10.1038/ s41401-021-00613-8, published online 24 Feb 2021

The author order and affiliation should be corrected to

Ya-jin Liao ${ }^{1,2,3,4 \#^{*}}$, Rui-yuan Pan ${ }^{2 \#}$, Xiang-xi Kong ${ }^{2 \#, ~ Y o n g ~}$ Cheng ${ }^{1}$, Lu Du ${ }^{2}$, Zhen-Chao Wang ${ }^{3}$, Chao Yuan ${ }^{2}$, Jin-bo Cheng ${ }^{1}$, Zeng-qiang Yuan ${ }^{2}$, Hai-yan Zhang ${ }^{3^{*}}$

1. Center on Translational Neuroscience, College of Life and Environmental Sciences, Minzu University of China, Beijing 100081, China.

2. Brain Science Center, Beijing Institute of Basic Medical Sciences, Beijing 100850, China.

3. Key Laboratory of Modern Preparation of TCM, Ministry of Education, Jiangxi University of Traditional Chinese Medicine, Nanchang 330004, China.

4. NHC Key Laboratory of Birth Defects Research, Prevention and Treatment, Hunan Provincial Maternal and Child Health Care Hospital, Changsha 410005, China.

"These authors contributed equally to this work.

* Corresponding author:

Ya-jin Liao Email: lyajin@muc.edu.cn

Hai-yang Zhang Email: haiyansl@163.com

The authors apologize that the lane 1 of the pro-Casp- 1 band in Figure $1 \mathrm{~b}$ was missed due to our neglect when preparation of the manuscript, in the initially published version of this article.
The Figure $1 \mathrm{~b}$ is revised as below.

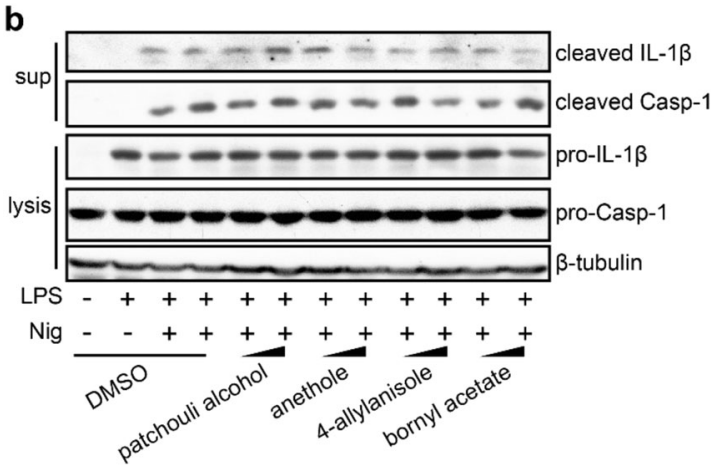

Below is the original picture of the pro-Casp-1 band of Figure $1 \mathrm{~b}$

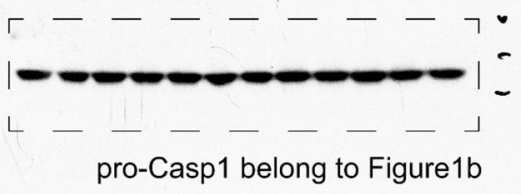

The authors apologize for any inconvenience caused to the journal and readers

${ }^{1}$ Center on Translational Neuroscience, College of Life and Environmental Sciences, Minzu University of China, Beijing 100081, China; ${ }^{2}$ Brain Science Center, Beijing Institute of Basic Medical Sciences, Beijing 100850, China; ${ }^{3}$ Key Laboratory of Modern Preparation of TCM, Ministry of Education, Jiangxi University of Traditional Chinese Medicine, Nanchang 330004, China and ${ }^{4}$ NHC Key Laboratory of Birth Defects Research, Prevention and Treatment, Hunan Provincial Maternal and Child Health Care Hospital, Changsha 410005, China Correspondence: Ya-jin Liao (lyajin@muc.edu.cn) or Hai-yan Zhang (haiyansl@163.com)

These authors contributed equally: Ya-jin Liao, Rui-yuan Pan, Xiang-xi Kong

Published online: 13 April 2021 